\title{
$E L R(+)$ chemokine signaling in host defense and disease in a viral model of central nervous system disease
}

\author{
Martin P. Hosking ${ }^{1 \dagger}$ and Thomas E. Lane ${ }^{2 *}$ \\ ' Department of Molecular Biology and Biochemistry, University of California, Irvine, CA, USA \\ ${ }^{2}$ Department of Pathology, Division of Microbiology and Immunology, School of Medicine, University of Utah, Salt Lake City, UT, USA
}

\section{Edited by:}

Richard M. Ransohoff, Cleveland

Clinic, USA

Reviewed by:

Stefano Pluchino, University of Cambridge, UK

Carolina Hoyo-Becerra, Clinic

Hospital Essen, Germany

*Correspondence:

Thomas E. Lane, Department of Pathology, Division of Microbiology and Immunology, School of

Medicine, University of Utah,

15 North Medical Drive East, Salt

Lake City, UT 84098, USA

e-mail: tom.lane@path.utah.edu

${ }^{\dagger}$ Present address:

Martin P. Hosking, Department of

Immunology and Microbial

Sciences, Scripps Research

Institute, La Jolla, CA 92037, USA
Intracranial infection of the neurotropic JHM strain of mouse hepatitis virus (JHMV) into the central nervous system (CNS) of susceptible strains of mice results in an acute encephalomyelitis, accompanied by viral replication in glial cells and robust infiltration of virus-specific $T$ cells that contribute to host defense through cytokine secretion and cytolytic activity. Mice surviving the acute stage of disease develop an immune-mediated demyelinating disease, characterized by viral persistence in white matter tracts and a chronic neuroinflammatory response dominated by $T$ cells and macrophages. Chemokines and their corresponding chemokine receptors are dynamically expressed throughout viral infection of the CNS, influencing neuroinflammation by regulating immune cell infltration and glial biology. This review is focused upon the pleiotropic chemokine receptor CXCR2 and its effects upon neutrophils and oligodendrocytes during JHMV infection and a number of other models of CNS inflammation.

Keywords: chemokines, chemokine receptors, virus, neuroinflammation, demyelination

\section{INTRODUCTION}

Intracranial infection of susceptible mice with the JHM strain of mouse hepatitis virus (JHMV) causes an acute encephalomyelitis followed by a chronic demyelinating disease. JHMV, after initially infecting ependymal cells lining the ventricles, rapidly disseminates to astrocytes, oligodendroglia, and microglia throughout the brain and spinal cord (Wang et al., 1992). Although inflammatory virus-specific $T$ cells are efficient in controlling viral replication through the secretion of IFN- $\gamma$ and cytolytic activity, sterile immunity is not achieved. Viral protein and/or RNA persist within oligodendroglia and drive continual $\mathrm{T}$ cell and macrophage infiltration, leading to chronic neuroinflammation and demyelination. Histological features associated with viral persistence include the development of an immune-mediated demyelinating disease similar to the human demyelinating disease MS; both T cells and macrophages are critical mediators of disease severity, contributing to myelin damage (Cheever et al., 1949; Perlman et al., 1999).

Through the course of acute and chronic JHMV-induced neurologic infection, there is a coordinated expression of chemokines and chemokine receptors that regulate inflammation, contributing to both host defense and disease exacerbation. Among the chemokines expressed during infection are members of the ELR $(+)$ chemokine family CXCL1 and CXCL2. CXCL1 and
CXCL2 are potent chemoattractants for peripheral mononuclear cells (PMNs), binding and signaling through their receptor CXCR2 (Wolpe et al., 1989; Moser et al., 1990; Schumacher et al., 1992; Marro et al., 2012; Weinger et al., 2013). Moreover, PMNs have been shown to enhance central nervous system (CNS) inflammation by disrupting blood brain barrier (BBB) integrity in animal models of spinal cord injury (SCI; Tonai et al., 2001; Gorio et al., 2007), autoimmune demyelination (Carlson et al., 2008), and JHMV-induced encephalomyelitis (Zhou et al., 2003), while blocking or silencing of CXCR2 signaling mutes inflammation and tissue damage in mouse models in which PMN infiltration is critical to disease initiation (Kielian et al., 2001; Belperio et al., 2005; Londhe et al., 2005a,b; Strieter et al., 2005; Gorio et al., 2007; Wareing et al., 2007; Carlson et al., 2008).

CXCR2 is also expressed by oligodendrocytes (Omari et al., 2005), and CXCL1 promotes the proliferation and positional migration of oligodendrocyte precursor cells (Robinson et al., 1998; Robinson and Franic, 2001; Tsai et al., 2002; Filipovic and Zecevic, 2008). Further, both CXCR2 and CXCL1 are expressed within active MS lesions (Omari et al., 2005, 2006). How and whether CXCR2 and its cognate ligands regulate immune and glial cell function during acute and chronic disease of the CNS is the focus of this review. 


\section{ELR(+) CHEMOKINE SIGNALING PROMOTES PMN INFILTRATION INTO THE CNS DURING ACUTE JHMV INFECTION}

Following JHMV infection, mRNA for the chemokine receptor CXCR2 and its associated ligands CXCL1 and CXCL2 are significantly upregulated within the acutely infected CNS, peaking at 3 days pi (Figure 1A). CXCL1 expression was localized to astrocytes (GFAP-positive) within the parenchyma and associated with the microvasculature (Figure 1B), consistent with previous observations (Lane et al., 1998; Omari et al., 2006; Rubio and Sanz-Rodriguez, 2007). The expression of the CXCR2 ligands within the CNS closely paralleled neutrophil emergency release into the circulation and infiltration into the CNS; CXCR2-expressing neutrophils were detectable as early as 1 day pi and peaked at 3 days pi within both the periphery and the CNS (Hosking et al., 2009).

To determine whether CXCR2 - signaling controlled neutrophil infiltration into the CNS, JHMV-infected mice were treated with either CXCR2 antiserum or control serum (NRS). Neutralization of CXCR2 almost completely abrogated neutrophil infiltration into the CNS (Figures 1C,D). Without infiltrating neutrophils, permeabilization of the blood-brain barrier was impaired (Hosking et al., 2009) and subsequent inflammatory cell infiltration was significantly reduced. Mice treated with CXCR2 neutralizing antiserum were incapable of controlling viral replication, and $100 \%$ of all infected mice succumbed to viral infection within 11 days and this was associated with an impaired ability to control CNS viral replication (Figures 1E,F). Moreover, total and virus specific $\mathrm{CD}^{+}$and $\mathrm{CD}^{+} \mathrm{T}$ cell infiltration into the CNS was diminished. Notably, CXCR2 neutralization did not alter the peripheral generation of virus-specific $\mathrm{T}$ cells, indicating that the increased mortality and diminished ability to control viral infection within the CNS is likely associated with the dampened access of $\mathrm{T}$ cells into the CNS parenchyma (Hosking et al., 2009). Collectively, these data demonstrate that during viral infection of the CNS, CXCR2 and its associated chemokines function to nonredundantly attract neutrophils into the CNS, where they are required to permeabilize the blood-brain barrier, thus facilitating subsequent inflammatory cell infiltration and control of viral replication.

\section{ELR(+) CHEMOKINE SIGNALING AND NEUTROPHILS IN OTHER MODELS OF CNS INFLAMMATION}

Neutrophils are amongst the earliest inflammatory infiltrate into the CNS following experimental autoimmune encephalitis (EAE) induction, and their presence precedes axonal damage, demyelination, and clinical disease (Carlson et al., 2008; Soulika et al., 2009; Wu et al., 2010). Neutralization of either CXCR2 (Carlson et al., 2008) or CXCL1 (Roy et al., 2012) potently reduces neutrophil infiltration into the CNS and reduces BBB permeability, thereby significantly delaying the onset and peak of clinical symptoms. Neutrophils also infiltrate into the CNS during the first week following cuprizone feeding, and their early presence in the CNS is absolutely necessary for the subsequent demyelination observed within the corpus callosum (Liu et al., 2010a). CXCR2 deficient mice or bone marrow chimeric mice, where myeloid cells lack CXCR2, or neutrophildepleted mice are resistant to cuprizone induced demyelination (Liu et al., 2010a). Interestingly, although neutrophils are also critical for lymphocytic choriomeningitis virus (LCMV)and pilocarpine-induced BBB permeabilization and subsequent seizures (Fabene et al., 2008; Kim et al., 2009), they are dispensable for seizures during Theiler's murine encephalomyelitis virus (TMEV; Libbey et al., 2011), underlining the fact that neutrophils are not the only cell type capable of mediating permeabilizing the $\mathrm{BBB}$. To this point, resident monocytes, astrocytes, and $\mathrm{CD}^{+} \mathrm{T}$ cells are all capable of direct permeabilization (Savarin et al., 2010, 2011; Johnson et al., 2012). Nevertheless, CXCR2-directed neutrophil infiltration into the CNS is a key determinate for subsequent inflammatory cell infiltration in a variety of CNS models of viral infection, demyelination, and autoimmunity.

\section{ELR(+) CHEMOKINE SIGNALING PROMOTES OLIGODENDROGLIA SURVIVAL DURING CHRONIC JHMV-INDUCED DEMYELINATION}

How chemokine receptor signaling contributes to chronic neurologic diseases has largely been considered within the context of targeted leukocyte recruitment into the CNS (Liu et al., 2000, 2001a,b; Glass and Lane, 2003; Hosking et al., 2009). However, numerous resident cell types of the CNS also express chemokine receptors under non-inflammatory and inflammatory conditions (reviewed in Bajetto et al., 2001; Ubogu et al., 2006), indicating that these cells are capable of responding to specific chemokine ligands. Thus, chemokine signaling may participate in either repair and/or exacerbation of pathology following insult, injury, or infection of the CNS (Liu et al., 2001b; Kerstetter et al., 2009; Omari et al., 2009).

Following JHMV infection, mRNA transcripts for CXCR2 as well as its ligands CXCL1 and CXCL2 are significantly upregulated, persisting until at least 21 days pi within the spinal cord (Figure 2A). CXCL1 expression was localized to GFAP+ astrocytes within the white matter (Figure 2B), suggesting that CXCR2, besides attracting neutrophils during early acute viral infection, may also alternatively function during chronic demyelination. To determine whether CXCR2 signaling was beneficial or pathogenic, mice persistently infected with JHMV were treated with anti-CXCR2 or control serum (NRS) from day 12-20 p.i. CXCR2 neutralization significantly delayed spontaneous clinical recovery (Figure 2C). Correspondingly, spinal cords from anti-CXCR2 treated mice revealed significantly greater areas of demyelination (Figures 2D,E). Importantly, CXCR2 neutralization during chronic JHMV infection did not affect inflammatory cell infiltration into the CNS (Hosking et al., 2010).

CXCR2 neutralization was also associated with an increase of apoptotic oligodendrocytes and oligodendrocyte precursor cells within white matter tracts of the spinal cord (Figure 2F; Hosking et al., 2010). To determine whether or not CXCR2 


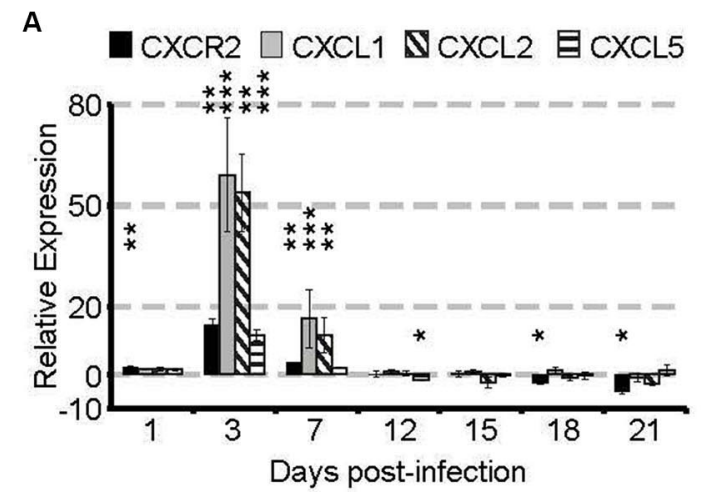

C

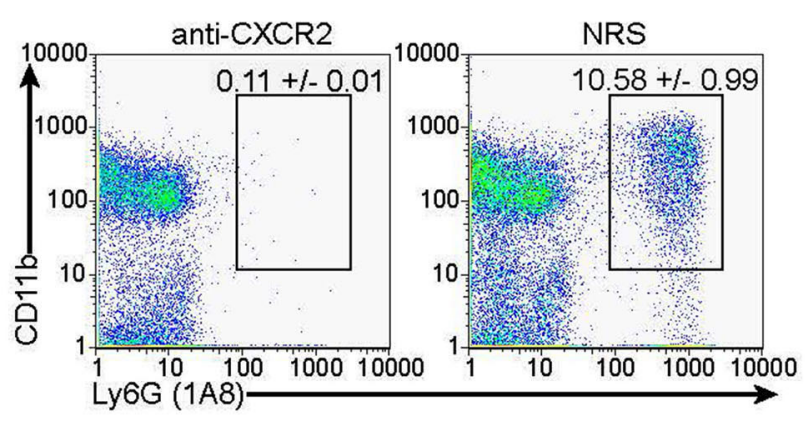

E

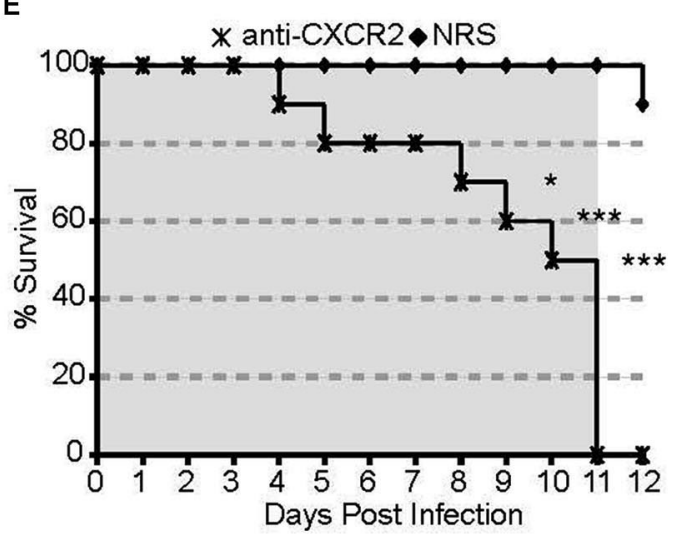

FIGURE 1 | CXCR2 drives neutrophil infiltration into the CNS during acute JHMV infection. C57BL/6 mice were infected with JHMV and their brains removed at the indicated time points. (A) mRNA for CXCR2, CXCL1 and CXCL2 are upregulated within the brains of JHMV infected mice. (B) Immunofluorescence staining reveals that the majority of CXCL1 (green) co-localizes with GFAP+ (red) astrocytes. (C) Representative FACS plots
B

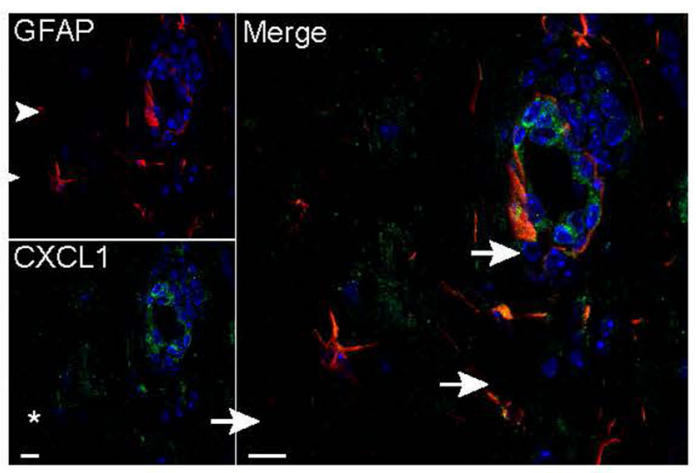

D
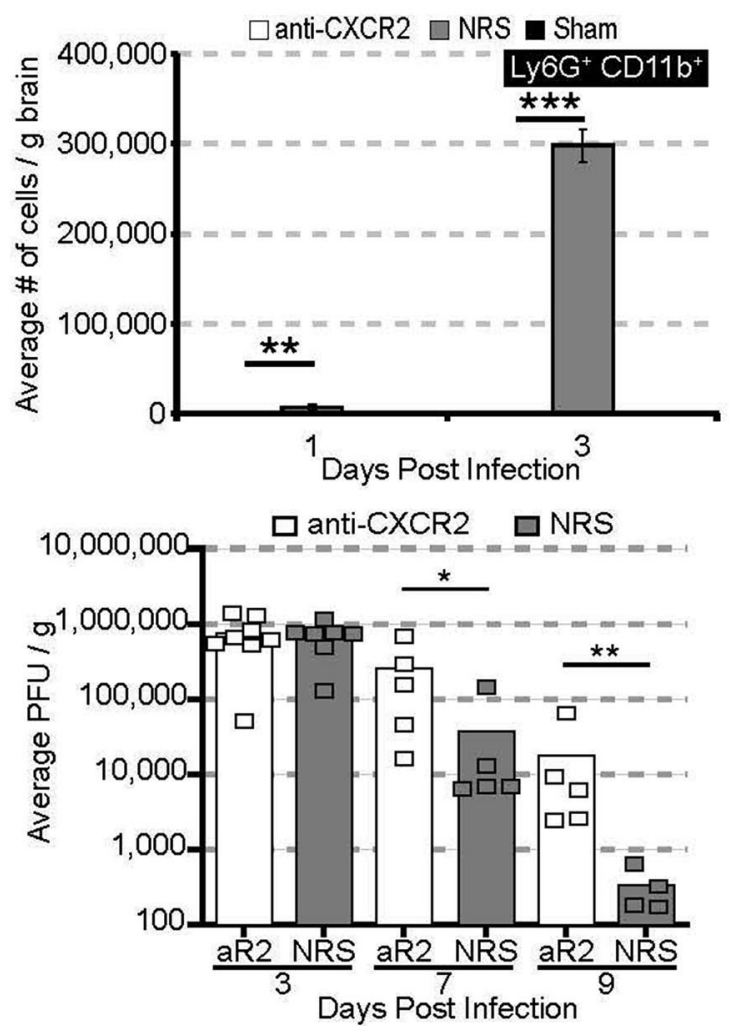

depicting the average frequency of neutrophils at day 3 are shown in panel. (D) Neutralization of CXCR2 blocks neutrophil (Ly6G ${ }^{+}$CD11 b ${ }^{+}$) infiltration into the CNS. (E) CXCR2 neutralization results in 100\% morality by day 11 pi (shaded area indicates the treatment period) and (F) elevated viral loads within the brains of treated mice. NRS $=$ normal rabbit serum treated mice. ${ }^{*} p<0.05,{ }^{*} p<0.01,{ }^{*} *{ }^{*} p<0.001$ compared to NRS-treated mice. could directly prevent JHMV-mediated apoptosis, cultured oligodendroglia were infected with JHMV in vitro and treated with varying concentrations of CXCL1. In accordance with previous observations (Liu et al., 2003, 2006; Liu and Zhang, 2005, 2007), JHMV-infected oligodendrocytes readily underwent apoptosis (Figure 2G), and western blotting confirmed activated caspase 3, cleaved poly ADP ribose polymerase (PARP) (a caspase 3 target), and muted expression of Bcl-2 (Figure 2I). CXCL1, in a dose-dependent manner, prevented JHMV-mediated apoptosis (Figure 2G). Moreover, activated caspase 3 and cleaved PARP were undetectable in CXCL1-treated cultures (Figure 2I). Notably, CXCL1 was incapable of rescuing CXCR2 deficient cultures from JHMV-mediated apoptosis (Figures 2H,I). CXCR2 also prevents IFN $\gamma$-and CXCL10- mediated apoptosis of murine or human oligodendroglia cultures (Tirotta et al., 2011, 2012). Collectively, these data suggest that CXCR2, during chronic viral infection of the CNS, prevents oligodendrocyte 


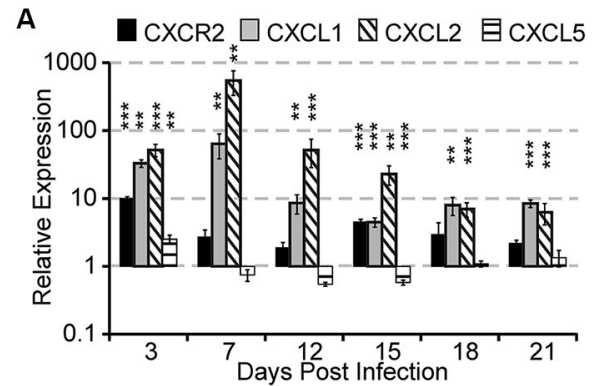

C

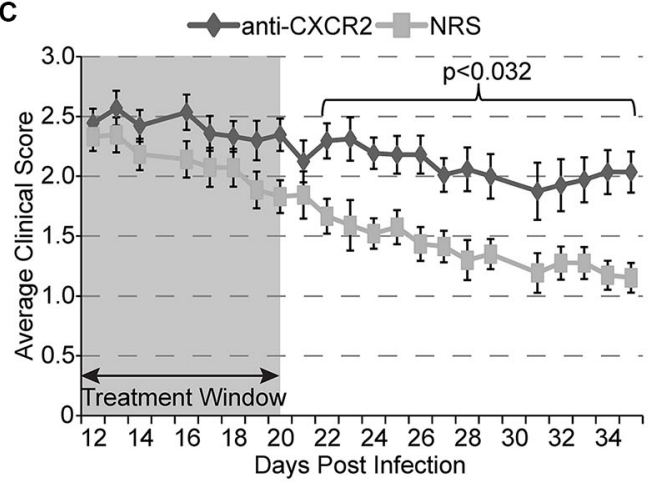

E

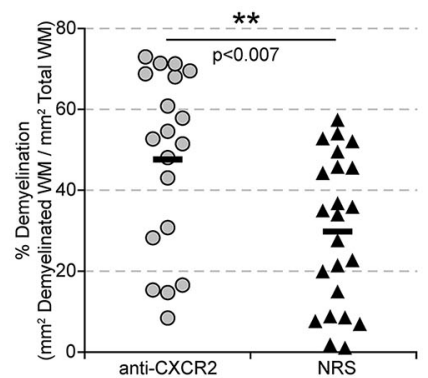

$\mathrm{H}$

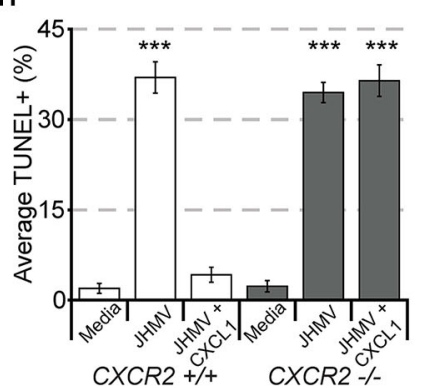

I

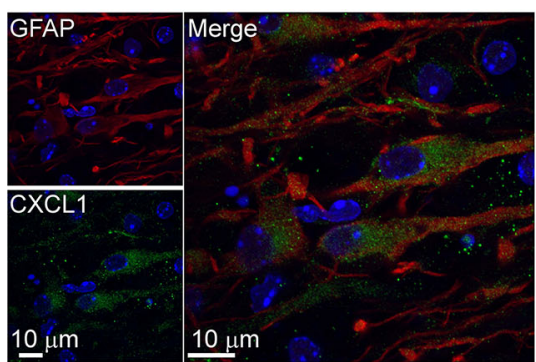

D

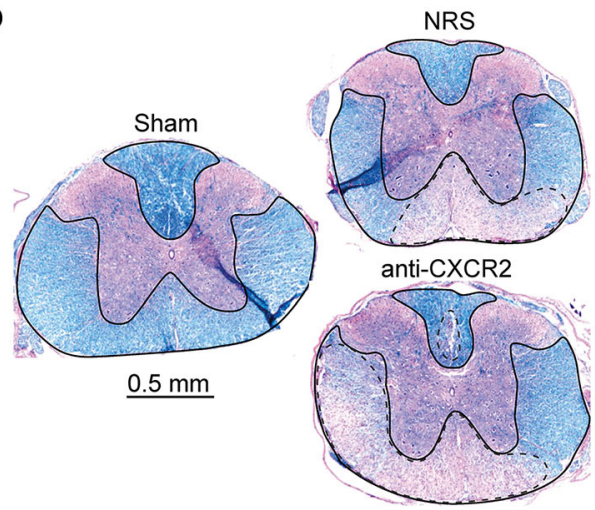

G
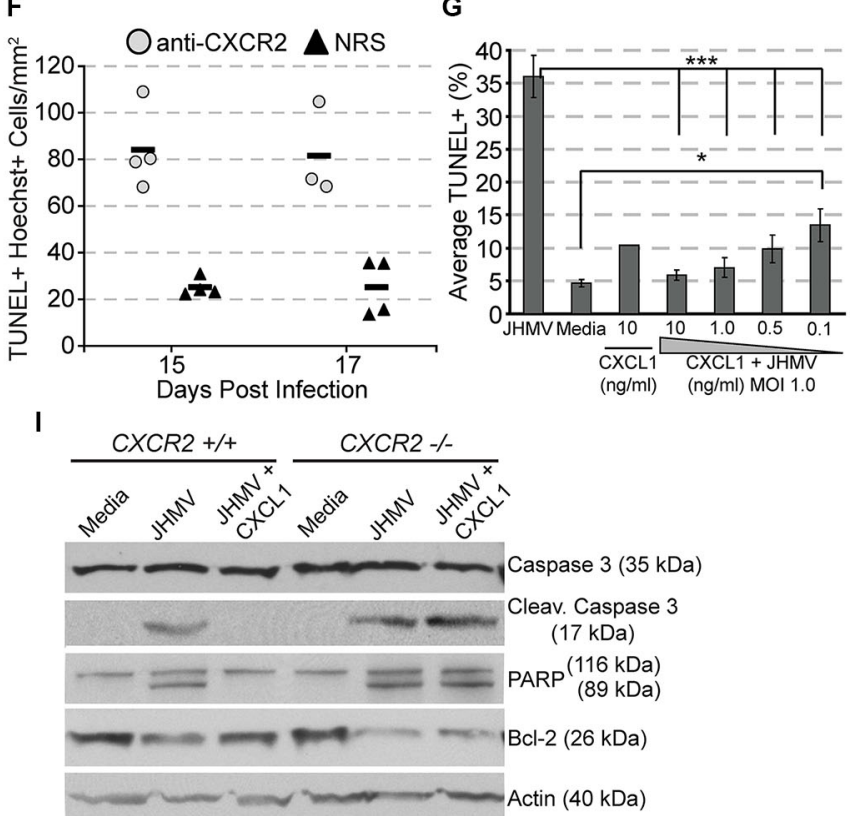

FIGURE 2 | CXCR2 promotes spontaneous recovery and oligodendrocyte survival during chronic JHMV infection. C57BL/6 mice were infected with JHMV and their spinal cords removed at the indicated time points. (A) mRNA for CXCR2, CXCL1, and CXCL2 are upregulated within the spinal cords of JHMV infected mice. (B) Immunofluorescence staining reveals that the majority of CXCL1 (green) co-localizes with GFAP-positive (red) astrocytes within the spinal cord white matter. (C) Neutralization of CXCR2 (from day 12-20 pi) delays clinical recovery from chronic JHMV infection. (D and E) Mice receiving CXCR2 antiserum had significantly greater total areas of demyelination within the spinal cord. Representative luxol fast blue stained spinal cords are shown in panel (D) with the total (solid line) and demyelinated (dashed line) white matter indicated. (F) Significantly $(p<0.001)$ increased numbers of apoptotic (TUNEL+) cells were observed within the spinal cords of anti-CXCR2 treated mice. (G) CXCL1, in a dose-dependent manner, protects oligodendrocytes from apoptosis, and (H) CXCR2-deficienct oligodendrocyte-enriched cultures are not protected from apoptosis. (I) Protein lysates from CXCR2-sufficient and CXCR2-deficient oligodendrocyte cultures were assessed via western blot for total caspase 3, activated caspase 3, PARP, Bcl-2, and actin expression. NRS $=$ normal rabbit serum treated mice. ${ }^{*} p<0.05,{ }^{*} p<$ $0.01,{ }^{* * *} p<0.001$ compared to NRS-treated mice. 
apoptosis and promotes clinical recovery from viral induced demyelination.

\section{ELR(+) CHEMOKINE SIGNALING AND OTHER MODELS OF CNS INFLAMMATION AND DEMYELINATION}

The role for CXCR2 signaling during EAE and a variety of toxin-induced demyelination models has also been studied. Raine and colleagues (Omari et al., 2009) have shown that CXCL1, when inducibly expressed by astrocytes after the onset of EAE, reduces peak disease severity, reduces total demyelination, and increases the onset of remyelination. Moreover, transgenic CXCL1 was associated with greater proliferation (presumably of oligodendrocyte precursors) throughout the spinal cord white matter (Omari et al., 2009). Conversely, Ransohoff and colleagues (Liu et al., 2010b) have demonstrated, using a series of bone marrow chimeras, that parenchymal CXCR2 deficiency on radio-resistant cells promotes faster recovery from EAE, cuprizone-induced demyelination, and in vitro lysotecithin-induced demyelination. Notably, initial clinical severity, inflammation, and/or demyelination in all three models of demyelination and repair were similar regardless of whether parenchymal cells possessed CXCR2; accelerated recovery was associated with initial increases in oligodendrocyte precursor cells, followed by an increased density of mature myelinating oligodendrocytes (Liu et al., 2010b). Similar results were observed following CXCR2 chemical anatagonism during EAE and in vivo lysolecithin-induced demyelination (Kerstetter et al., 2009).

\section{PERSPECTIVES}

The JHMV-induced model of viral-induced encephalomyelitis provides an important tool in defining molecular and cellular mechanisms that regulate neuroinflammation during both host defense and disease progression. Our research on chemokines and chemokine receptors has revealed important roles for these molecules in orchestrating CNS inflammation in response to JHMV infection. We and others have found unique and pleiotropic roles for ELR+ chemokine signaling via CXCR2 in moderating neutrophil infiltration and protecting oligodendroglia from apoptosis in response to exposure to virus and proinflammatory cytokines. Ongoing research in our laboratory continues to focus on the role of $\operatorname{ELR}(+)$ chemokine signaling on oligodendroglia during JHMV-induced neuroinflammation. It will be important to analyze the effects of selectively ablating CXCR2 on oligodendroglia during JHMV-induced demyelination, while simultaneously manipulating the cellular sources of ELR-positive chemokines in the CNS that may promote neuroprotection during chronic JHMV-induced disease.

\section{ACKNOWLEDGMENTS}

This work was funded by National Institutes of Health (NIH) Grant R01 NS41249 to Thomas E. Lane and T32 HL007195-34 to Martin P. Hosking.

\section{REFERENCES}

Bajetto, A., Bonavia, R., Barbero, S., Florio, T., and Schettini, G. (2001). Chemokines and their receptors in the central nervous system. Front. Neuroendocrinol. 22, 147-184. doi: 10.1006/frne.2001.0214
Belperio, J. A., Keane, M. P., Burdick, M. D., Gomperts, B. N., Xue, Y. Y., Hong, K., et al. (2005). CXCR2/CXCR2 ligand biology during lung transplant ischemiareperfusion injury. J. Immunol. 175, 6931-6939. doi: 10.4049/jimmunol.175.10. 6931

Carlson, T., Kroenke, M., Rao, P., Lane, T. E., and Segal, B. (2008). The Th17-ELR+ CXC chemokine pathway is essential for the development of central nervous system autoimmune disease. J. Exp. Med. 205, 811-823. doi: 10.1084/jem. 20072404

Cheever, F. S., Daniels, J. B., Pappenheimer, A. M., and Bailey, O. T. (1949). A murine virus (JHM) causing disseminated encephalomyelitis with extensive destruction of myelin. J. Exp. Med. 90, 181-194. doi: 10.1084/jem. 90.3.181

Fabene, P. F., Navarro Mora, G., Martinello, M., Rossi, B., Merigo, F., Ottoboni, L., et al. (2008). A role for leukocyte-endothelial adhesion mechanisms in epilepsy. Nat. Med. 14, 1377-1383. doi: 10.1038/nm.1878

Filipovic, R., and Zecevic, N. (2008). The effect of CXCL1 on human fetal oligodendrocyte progenitor cells. Glia 56, 1-15. doi: 10.1002/glia.20582

Glass, W. G., and Lane, T. E. (2003). Functional expression of chemokine receptor CCR5 on CD4(+) T cells during virus-induced central nervous system disease. J. Virol. 77, 191-198. doi: 10.1128/jvi.77.1.191-198.2003

Gorio, A., Madaschi, L., Zadra, G., Marfia, G., Cavalieri, B., Bertini, R., et al. (2007). Reparixin, an inhibitor of CXCR2 function, attenuates inflammatory responses and promotes recovery of function after traumatic lesion to the spinal cord. J. Pharmacol. Exp. Ther. 322, 973-981. doi: 10.1124/jpet.107. 123679

Hosking, M. P., Liu, L., Ransohoff, R. M., and Lane, T. E. (2009). A protective role for ELR+ chemokines during acute viral encephalomyelitis. PLoS Pathog. 5:e1000648. doi: 10.1371/journal.ppat.1000648

Hosking, M. P., Tirotta, E., Ransohoff, R. M., and Lane, T. E. (2010). CXCR2 signaling protects oligodendrocytes and restricts demyelination in a mouse model of viral-induced demyelination. PLoS One 5:e11340. doi: 10.1371/journal.pone. 0011340

Johnson, H. L., Chen, Y., Jin, F., Hanson, L. M., Gamez, J. D., Pirko, I., et al. (2012). CD8 T cell-initiated blood-brain barrier disruption is independent of neutrophil support. J. Immunol. 189, 1937-1945. doi: 10.4049/jimmunol.12 00658

Kerstetter, A. E., Padovani-Claudio, D. A., Bai, L., and Miller, R. H. (2009). Inhibition of CXCR2 signaling promotes recovery in models of multiple sclerosis. Exp. Neurol. 220, 44-56. doi: 10.1016/j.expneurol.2009.07.010

Kielian, T., Barry, B., and Hickey, W. F. (2001). CXC chemokine receptor-2 ligands are required for neutrophil-mediated host defense in experimental brain abscesses. J. Immunol. 166, 4634-4643. doi: 10.4049/jimmunol.166.7.4634

Kim, J. V., Kang, S. S., Dustin, M. L., and Mcgavern, D. B. (2009). Myelomonocytic cell recruitment causes fatal CNS vascular injury during acute viral meningitis. Nature 457, 191-195. doi: 10.1038/nature07591

Lane, T. E., Asensio, V. C., Yu, N., Paoletti, A. D., Campbell, I. L., and Buchmeier, M. J. (1998). Dynamic regulation of alpha- and beta-chemokine expression in the central nervous system during mouse hepatitis virus-induced demyelinating disease. J. Immunol. 160, 970-978.

Libbey, J. E., Kennett, N. J., Wilcox, K. S., White, H. S., and Fujinami, R. S. (2011). Interleukin-6, produced by resident cells of the central nervous system and infiltrating cells, contributes to the development of seizures following viral infection. J. Virol. 85, 6913-6922. doi: 10.1128/JVI.00458-11

Liu, L., Belkadi, A., Darnall, L., Hu, T., Drescher, C., Cotleur, A. C., et al. (2010a). CXCR2-positive neutrophils are essential for cuprizone-induced demyelination: relevance to multiple sclerosis. Nat. Neurosci. 13, 319-326. doi: 10.1038/ nn. 2491

Liu, L., Darnall, L., Hu, T., Choi, K., Lane, T. E., and Ransohoff, R. M. (2010b). Myelin repair is accelerated by inactivating CXCR2 on nonhematopoietic cells. J. Neurosci. 30, 9074-9083. doi: 10.1523/JNEUROSCI.1238-10.2010

Liu, M. T., Armstrong, D., Hamilton, T. A., and Lane, T. E. (2001a). Expression of Mig (monokine induced by interferon-gamma) is important in T lymphocyte recruitment and host defense following viral infection of the central nervous system. J. Immunol. 166, 1790-1795. doi: 10.4049/jimmunol.166. 3.1790

Liu, M. T., Chen, B. P., Oertel, P., Buchmeier, M. J., Armstrong, D., Hamilton, T. A., et al. (2000). The T cell chemoattractant IFN-inducible protein 10 is essential in host defense against viral-induced neurologic disease. J. Immunol. 165, 2327 2330. doi: 10.4049/jimmunol.165.5.2327 
Liu, M. T., Keirstead, H. S., and Lane, T. E. (2001b). Neutralization of the chemokine CXCL10 reduces inflammatory cell invasion and demyelination and improves neurological function in a viral model of multiple sclerosis. J. Immunol. 167, 4091-4097. doi: 10.4049/jimmunol.167.7.4091

Liu, Y., and Zhang, X. (2005). Expression of cellular oncogene Bcl-xL prevents coronavirus-induced cell death and converts acute infection to persistent infection in progenitor rat oligodendrocytes. J. Virol. 79, 47-56. doi: 10.1128/jvi.79. 1.47-56.2005

Liu, Y., and Zhang, X. (2007). Murine coronavirus-induced oligodendrocyte apoptosis is mediated through the activation of the Fas signaling pathway. Virology 360, 364-375. doi: 10.1016/j.virol.2006.10.044

Liu, Y., Cai, Y., and Zhang, X. (2003). Induction of caspase-dependent apoptosis in cultured rat oligodendrocytes by murine coronavirus is mediated during cell entry and does not require virus replication. J. Virol. 77, 11952-11963. doi: 10. 1128/jvi.77.22.11952-11963.2003

Liu, Y., Pu, Y., and Zhang, X. (2006). Role of the mitochondrial signaling pathway in murine coronavirus-induced oligodendrocyte apoptosis. J. Virol. 80, 395-403. doi: 10.1128/jvi.80.1.395-403.2006

Londhe, V. A., Belperio, J. A., Keane, M. P., Burdick, M. D., Xue, Y. Y., and Strieter, R. M. (2005a). CXCR2 is critical for dsRNA-induced lung injury: relevance to viral lung infection. J. Inflamm. (Lond). 2:4. doi: 10.1186/14769255-2-4

Londhe, V. A., Belperio, J. A., Keane, M. P., Burdick, M. D., Xue, Y. Y., and Strieter, R. M. (2005b). CXCR2/CXCR2 ligand biological axis impairs alveologenesis during dsRNA-induced lung inflammation in mice. Pediatr. Res. 58, 919-926. doi: 10.1203/01.pdr.0000181377.78061.3e

Marro, B. S., Hosking, M. P., and Lane, T. E. (2012). CXCR2 signaling and host defense following coronavirus-induced encephalomyelitis. Future Virol. 7, 349359. doi: $10.2217 /$ fvl.12.23

Moser, B., Clark-Lewis, I., Zwahlen, R., and Baggiolini, M. (1990). Neutrophilactivating properties of the melanoma growth-stimulatory activity. J. Exp. Med. 171, 1797-1802. doi: 10.1084/jem.171.5.1797

Omari, K. M., John, G., Lango, R., and Raine, C. S. (2006). Role for CXCR2 and CXCL1 on glia in multiple sclerosis. Glia 53, 24-31. doi: 10.1002/glia.20246

Omari, K. M., John, G. R., Sealfon, S. C., and Raine, C. S. (2005). CXC chemokine receptors on human oligodendrocytes: implications for multiple sclerosis. Brain 128, 1003-1015. doi: 10.1093/brain/awh479

Omari, K. M., Lutz, S. E., Santambrogio, L., Lira, S. A., and Raine, C. S. (2009). Neuroprotection and remyelination after autoimmune demyelination in mice that inducibly overexpress CXCL1. Am. J. Pathol. 174, 164-176. doi: 10.2353/ajpath. 2009.080350

Perlman, S. R., Lane, T. E., and Buchmeier, M. J. (1999). "Coronaviruses: hepatitis, peritonitis and central nervous system disease," in Effects of Microbes on the Immune System, eds M. W. Cunningham and R. S. Fujinami (Philadelphia: Lippincott Williams and Wilkins), 331-348.

Robinson, S., and Franic, L. A. (2001). Chemokine GRO1 and the spatial and temporal regulation of oligodendrocyte precursor proliferation. Dev. Neurosci. 23, 338-345. doi: 10.1159/000048717

Robinson, S., Tani, M., Strieter, R. M., Ransohoff, R. M., and Miller, R. H. (1998). The chemokine growth-regulated oncogene-alpha promotes spinal cord oligodendrocyte precursor proliferation. J. Neurosci. 18, 10457-10463.

Roy, M., Richard, J. F., Dumas, A., and Vallieres, L. (2012). CXCL1 can be regulated by IL- 6 and promotes granulocyte adhesion to brain capillaries during bacterial toxin exposure and encephalomyelitis. J. Neuroinflammation 9:18. doi: 10. 1186/1742-2094-9-18

Rubio, N., and Sanz-Rodriguez, F. (2007). Induction of the CXCL1 (KC) chemokine in mouse astrocytes by infection with the murine encephalomyelitis virus of Theiler. Virology 358, 98-108. doi: 10.1016/j.virol.2006.08.003

Savarin, C., Stohlman, S. A., Atkinson, R., Ransohoff, R. M., and Bergmann, C. C. (2010). Monocytes regulate $\mathrm{T}$ cell migration through the glia limitans during acute viral encephalitis. J. Virol. 84, 4878-4888. doi: 10.1128/JVI. 00051-10

Savarin, C., Stohlman, S. A., Rietsch, A. M., Butchi, N., Ransohoff, R. M., and Bergmann, C. C. (2011). MMP9 deficiency does not decrease blood-brain barrier disruption, but increases astrocyte MMP3 expression during viral encephalomyelitis. Glia 59, 1770-1781. doi: 10.1002/glia.21222
Schumacher, C., Clark-Lewis, I., Baggiolini, M., and Moser, B. (1992). Highand low-affinity binding of GRO alpha and neutrophil-activating peptide 2 to interleukin 8 receptors on human neutrophils. Proc. Natl. Acad. Sci. U S A 89, 10542-10546. doi: 10.1073/pnas.89.21.10542

Soulika, A. M., Lee, E., Mccauley, E., Miers, L., Bannerman, P., and Pleasure, D. (2009). Initiation and progression of axonopathy in experimental autoimmune encephalomyelitis. J. Neurosci. 29, 14965-14979. doi: 10.1523/JNEUROSCI. 3794-09.2009

Strieter, R. M., Keane, M. P., Burdick, M. D., Sakkour, A., Murray, L. A., and Belperio, J. A. (2005). The role of CXCR2/CXCR2 ligands in acute lung injury. Curr. Drug Targets Inflamm. Allergy 4, 299-303. doi: 10.2174/15680100540 22178

Tirotta, E., Kirby, L. A., Hatch, M. N., and Lane, T. E. (2012). IFN-gamma-induced apoptosis of human embryonic stem cell derived oligodendrocyte progenitor cells is restricted by CXCR2 signaling. Stem Cell Res. 9, 208-217. doi: 10.1016/j. scr.2012.06.005

Tirotta, E., Ransohoff, R. M., and Lane, T. E. (2011). CXCR2 signaling protects oligodendrocyte progenitor cells from IFN-gamma/CXCL10-mediated apoptosis. Glia 59, 1518-1528. doi: 10.1002/glia.21195

Tonai, T., Shiba, K., Taketani, Y., Ohmoto, Y., Murata, K., Muraguchi, M., et al. (2001). A neutrophil elastase inhibitor (ONO-5046) reduces neurologic damage after spinal cord injury in rats. J. Neurochem. 78, 1064-1072. doi: 10.1046/j. 1471-4159.2001.00488.x

Tsai, H. H., Frost, E., To, V., Robinson, S., Ffrench-Constant, C., Geertman, R., et al. (2002). The chemokine receptor CXCR2 controls positioning of oligodendrocyte precursors in developing spinal cord by arresting their migration. Cell 110, 373-383. doi: 10.1016/s0092-8674(02)00838-3

Ubogu, E. E., Cossoy, M. B., and Ransohoff, R. M. (2006). The expression and function of chemokines involved in CNS inflammation. Trends Pharmacol. Sci. 27, 48-55. doi: 10.1016/j.tips.2005.11.002

Wang, F. I., Hinton, D. R., Gilmore, W., Trousdale, M. D., and Fleming, J. O. (1992). Sequential infection of glial cells by the murine hepatitis virus JHM strain (MHV-4) leads to a characteristic distribution of demyelination. Lab. Invest. 66, 744-754.

Wareing, M. D., Shea, A. L., Inglis, C. A., Dias, P. B., and Sarawar, S. R. (2007). CXCR2 is required for neutrophil recruitment to the lung during influenza virus infection, but is not essential for viral clearance. Viral Immunol. 20, 369-378. doi: 10.1089/vim.2006.0101

Weinger, J. G., Marro, B. S., Hosking, M. P., and Lane, T. E. (2013). The chemokine receptor CXCR2 and coronavirus-induced neurologic disease. Virology 435, 110-117. doi: 10.1016/j.virol.2012.08.049

Wolpe, S. D., Sherry, B., Juers, D., Davatelis, G., Yurt, R. W., and Cerami, A. (1989). Identification and characterization of macrophage inflammatory protein 2. Proc. Natl. Acad. Sci. U S A 86, 612-616. doi: 10.1073/pnas.86.2.612

Wu, F., Cao, W., Yang, Y., and Liu, A. (2010). Extensive infiltration of neutrophils in the acute phase of experimental autoimmune encephalomyelitis in C57BL/6 mice. Histochem. Cell Biol. 133, 313-322. doi: 10.1007/s00418-009-0673-2

Zhou, J., Stohlman, S. A., Hinton, D. R., and Marten, N. W. (2003). Neutrophils promote mononuclear cell infiltration during viral-induced encephalitis. J. Immunol. 170, 3331-3336. doi: 10.4049/jimmunol.170.6.3331

Conflict of Interest Statement: The authors declare that the research was conducted in the absence of any commercial or financial relationships that could be construed as a potential conflict of interest.

Received: 06 March 2014; accepted: 31 May 2014; published online: 17 June 2014. Citation: Hosking MP and Lane TE (2014) ELR(+) chemokine signaling in host defense and disease in a viral model of central nervous system disease. Front. Cell. Neurosci. 8:165. doi: 10.3389/fncel.2014.00165

This article was submitted to the journal Frontiers in Cellular Neuroscience.

Copyright (C) 2014 Hosking and Lane. This is an open-access article distributed under the terms of the Creative Commons Attribution License (CC BY). The use, distribution or reproduction in other forums is permitted, provided the original author(s) or licensor are credited and that the original publication in this journal is cited, in accordance with accepted academic practice. No use, distribution or reproduction is permitted which does not comply with these terms. 